1 Black bib size is associated with feather content of pheomelanin in male 2 house sparrows

3 Ismael Galván ${ }^{1, *}$, Kazumasa Wakamatsu ${ }^{2}$ and Carlos Alonso-Alvarez ${ }^{3}$

5 1. Departamento de Ecología Evolutiva, Estación Biológica de Doñana - CSIC, c/ Américo Vespucio s/n, 641092 Sevilla, Spain.

2. Department of Chemistry, Fujita Health University School of Health Sciences, Toyoake, Aichi 470-1192, 8 Japan.

3. Instituto de Investigación en Recursos Cinegéticos (IREC) - CSIC-UCLM-JCCM, Ronda de Toledo s/n, 13005 Ciudad Real, Spain.

*Corresponding author. Departamento de Ecología Evolutiva, Estación Biológica de Doñana - CSIC, c/ Américo Vespucio s/n, 41092 Sevilla, Spain, phone: +34 954466700, fax: +34 954621125, email: galvan@ebd.csic.es

Word count: 2173

Keywords: bib size, genotypic quality, house sparrow, pheomelanin, signal honesty

Running title: Melanin content of the bib of house sparrows. 
Dear Editor,

Pigments constitute the basis of most visual signals involved in animal communication. Melanins are the most common pigments, often representing the basis of signals that are honest (i.e. signalers transfer reliable information about their genotypic quality to receivers; McGraw, 2008). The black chest bib of male house sparrows Passer domesticus is a melanin-based plumage patch (figure 1) that constitutes one of the most intensively studied animal signals (Anderson, 2006). Birds develop the bib not only during the breeding season but all year round, larger bibs being likely associated with higher melanocyte numbers. Male house sparrows displaying larger bibs are dominant in aggressive interactions with other males, have better body condition, and, in some populations, achieve higher lifetime reproductive success (reviewed in Nakagawa et al., 2007). This has been explained through the handicap principle, i.e. low-quality house sparrows do not display large bibs because their production represents costs that these birds cannot afford. In particular, bib expression depends on testosterone levels which in turn suppresses the immune system, and immunosuppression costs would be only affordable by high-quality males (i.e., the immunocompetence handicap hypothesis; González et al., 1999).

However, the signal content of house sparrows' bibs is assumed to be intimately related to their constitutive pigments (i.e. melanins), so signal expression (i.e. bib size) is likely to depend more primarily on the mechanisms that control overall melanin biosynthesis than on testosterone level, although testosterone clearly affects the deposition of melanins in feathers (e.g., Haase et al., 1995). Two main chemically distinct forms of melanin are identified in vertebrate animals: eumelanin, a polymer of 5,6-dihydroxyindole (DHI) and 5,6-dihydroxyindole-2-carboxilic acid (DHICA) units, and pheomelanin, a polymer of benzothiazine and benzothiazole derivatives (García-Borrón and Olivares Sánchez, 2011). The environmental conditions under which producing pheomelanin and eumelanin is costly differ for each pigment (Galván and Alonso-Alvarez, 2009), and the genetic and physiological bases of melanogenesis are relatively well known (García-Borrón and Olivares Sánchez, 2011). Thus, understanding how the melanin composition of the house sparrow's bib affects its size might be very useful to understand why this signal is honest.

To our knowledge, a chemical analysis of house sparrow bib melanins has never been done, and it has been assumed that variation in bib characteristics depends on its content of 'melanin' in general terms or mostly on eumelanin content (McGraw, 2008). Thus, it has been assumed that the honesty of the signal should lie in producing more melanin (which would correspond to more melanocytes, assuming that melanin production per melanocyte is independent of bib size), but here we aim at determining the content of 
eumelanin and pheomelanin in bibs separately, considering that the mechanisms of synthesis of the two chemical forms differ and that this may help understanding the honesty of bib size. We therefore analyse the associations between the size of the bib of male house sparrows (figure 1) kept in captivity during the course of a experiment (in which the levels of the antioxidant glutathione, $\mathrm{GSH}$, were manipulated in some birds while others were controls) and the content of pheomelanin and eumelanin in their feathers. To measure pheomelanin, we used the specific markers 4-amino-3-hydroxyphenylalanine (4-AHP) and thiazole-2,4,5tricarboxylic acid (TTCA), and to analyse eumelanin, we used the specific marker pyrrole-2,3,5-tricarboxylic acid (PTCA) (see Online Supporting Information for experimental and analytical procedures).

The general linear model (GLM) exploring the relationship between final bib size (see Online Supporting Information) and TTCA concentration showed a negative and significant effect of TTCA levels $(b$ $=-6.02, t=-2.73, F_{1,36}=7.47, p=0.009$; figure 2$)$, in addition to the effects of experimental treatment $\left(F_{1,36}=\right.$ $3.75, p=0.061)$ and tarsus length $\left(F_{1,36}=0.01, p=0.933\right)$. The model with 4-AHP showed a marginally nonsignificant effect of this variable on bib size $\left(b=-2.09, t=-1.84, F_{1,37}=3.27, p=0.079\right)$. The relationship between bib size and PTCA levels was not significant $\left(b=1.13, F_{1,37}=0.16, p=0.686\right)$. Therefore, the bib size of male house sparrows was negatively correlated with the levels of pheomelanin (TTCA) in its feathers, and unrelated to the levels of eumelanin. The total content of eumelanin is considerably greater than the total content of pheomelanin but the latter is more variable among individual birds than the content of eumelanin (see Online Supporting Information).

Our results suggest that, in male house sparrows, developing large bibs means producing small amounts of pheomelanin. This may seem counterintuitive because some authors have assumed, but never proved, that bib size variability depends on eumelanin levels in the bib feathers as the black colour of the bib may be confered by eumelanin and not by pheomelanin, which produces lighter colours than eumelanin (McGraw, 2008). However, pheomelanin and eumelanin are produced under different conditions, and thiols that promote pheomelanin production inhibit the activity of the enzyme tyrosinase, hence reducing eumelanin production (del Marmol et al., 1993). Therefore, bib size may be either positively related to eumelanin levels or negatively related to pheomelanin levels. Our study confirms the latter. It also confirms previous observations suggesting that pheomelanin is biologically relevant even if present at low proportions in feathers whose appearance is given by the darker colors conferred by eumelanin (Galván et al., 2010). Recently, the size of a brown plumage patch in masked boobies Sula dactylatra has also been reported to be negatively associated to the levels of pheomelanin (4-AHP) but not related to the levels of eumelanin (PTCA) in the patch's feathers (Fargallo et al., 2014). Thus, the honesty in the signaling content of the black 
bib of male house sparrows should be based on the mechanisms that lead to the production of small amounts of one of its constitutive pigments, i.e. pheomelanin (see also Online Supporting Information).

This finding is relevant for understanding the honesty in the bib size of male house sparrows. The control of dishonesty might be mediated by the production costs of large bibs, and the only information on production costs so far proposed for this signaling system (although non-physiological costs are also possible for large plumage patches; Senar, 2006) is provided by the immunocompetence handicap hypothesis (González et al., 1999). However, to our knowledge there is no empirical demonstration that the physiological costs derived from testosterone-mediated immunosuppression are so high that the production of large bibs is not possible for low-quality birds (i.e., those with smaller bibs), just that males with large bibs have higher levels of immunosuppression (González et al., 1999). Therefore, production costs are not enough to explain why the signaling system is not invaded by cheaters producing bluffs, i.e. males producing bibs disproportionally larger relative to their genotypic quality, and the evolutionary logic of the honesty of house sparrows' bib remains unclear. Pheomelanin is produced when thiols (cysteine or GSH) are present, while eumelanin is produced when thiols are absent or below a threshold level in melanocytes (GarcíaBorrón and Olivares Sánchez, 2011). Therefore, to achieve a low production of pheomelanin that allows the development of a large bib it is necessary that thiol levels in melanocytes, where melanogenesis takes place, are low. In experiments not shown, we found that induced reductions of the levels of GSH led to the development of better phenotypes (i.e., larger bibs) in high-quality birds only (i.e., those which already displayed larger bibs before the experimental manipulation), suggesting that a physiological mechanism may have evolved in low-quality birds to avoid producing bibs of size not corresponding to their quality. Given the existence of specific transporters of cystine (the dimer of cysteine) that takes it into melanocytes (Chintala et al., 2005) or pump it out of melanosomes (Chiaverini et al., 2012), this may constitute a mechanism of control of signal expression. If the genetic basis of variation in the quality of male house sparrows was actually variation in the expression of genes coding for those transporters (SIc7a11 and CTNS; Chintala et al., 2005 and Chiaverini et al., 2012), then birds could develop bibs of size according to their quality and production costs would not be necessary to explain the honesty of the signal. That is, the expression of these genes may be linked to the quality of the birds so that the physiological state of low-quality birds may prevent them from producing large bibs even if those birds would decide to invest in the production of large bibs. Indeed, this opens a new perspective for the evolution of one of the most intensively studied systems of honest communication. 
116

117

118

119

120

121

122

123

124

125

126

127

128

129

130

131

132

133

134

135

136

137

138

139

140

\section{Acknowledgements}

This manuscript was greatly improved by the comments of Heinz Arnheiter and three anonymous reviewers. We thank the staff of Finca Dehesa Galiana (UCLM) where birds were housed. This study was funded by Consejería de Educación y Ciencia, Junta de Comunidades de Castilla la Mancha (project ref.: PII11090271-5037) and Ministerio de Economía y Competitividad (CGL2009-10883-C02-02 and CGL2012-40229C02-01) from the Spanish Government. I.G. benefited from a postdoctoral contract of the CSIC JAE-Doc program.

\section{References}

Anderson, T.R. 2006. Biology of the ubiquitous house sparrow (New York: Oxford University Press).

Chiaverini, C., Sillard, L., Flori, E., Ito, S., Briganti, S., Wakamatsu, K., Fontas, E., Berard, E., Cailliez, M., Cochat, P. et al. (2012). Cystinosin is a melanosomal protein that regulates melanin synthesis. FASEB J. 26, 3779-3789.

Chintala, S., Li, W., Lamoreux, M.L., Ito, S., Wakamatsu, K., Sviderskaya, E.V., Bennett, D.C., Park, Y.-M., Gahl, W.A., Huizing, M. et al. (2005). Slc7a11 gene controls production of pheomelanin pigment and proliferation of cultured cells. Proc. Natl. Acad. Sci. USA 102, 10964-10969.

del Marmol, V., Solano, F., Sels, A., Huez, G., Libert, A., Lejeune, F. and Ghanem, G. (1993). Glutathione depletion increases tyrosinase activity in human melanoma cells. J. Invest. Dermatol. 101, 871-874.

Fargallo, J.A., Velando, A., López-Rull, I., Gañán, N., Lifshitz, N., Wakamatsu, K. and Torres, R. (2014). Sexspecific phenotypic integration: endocrine profiles, coloration, and behavior in fledgling boobies. Behav. Ecol. 25, 76-87.

Galván, I. and Alonso-Alvarez, C. (2009). The expression of melanin-based plumage is separately modulated by exogenous oxidative stress and a melanocortin. Proc. R. Soc. B 276, 3089-3097.

Galván, I., Bijlsma, R.G., Negro, J.J., Jarén, M. and Garrido-Fernández, J. (2010). Environmental constraints for plumage melanization in the northern goshawk Accipiter gentilis. J. Avian Biol. 41, 523-531. 
García-Borrón, J.C. and Olivares Sánchez, M.C. (2011). Biosynthesis of melanins. In Melanins and melanosomes: biosynthesis, biogenesis, physiological, and pathological functions, J. Borovanský and P.A. Riley, eds. (Weinheim: Wiley-Blackwell), pp. 87-116.

González, G., Sorci, G. and de Lope, F. (1999). Seasonal variation in the relationship between cellular immune response and badge size in male house sparrows (Passer domesticus). Behav. Ecol. Sociobiol. 46, 117-122.

Haase, E., Ito, S. and Wakamatsu, K. (1995). Influences of sex, castration, and androgens on the eumelanin and pheomelanin contents of different feathers in wild mallards. Pigment Cell Res. 8, 164-170.

McGraw, K.J. (2008). An update on the honesty of melanin-based color signals in birds. Pigment Cell Melanoma Res. 21, 133-138.

Nakagawa, S., Ockendon, N., Gillespie, D.O.S., Hatchwell, B.J. and Burke, T. (2007). Assessing the function of house sparrows' bib size using a flexible meta-analysis method. Behav. Ecol. 18, 831-840.

Senar, J.C. (2006). Color displays as intrasexual signals of aggression and dominance. In Bird Coloration, volume 2: function and evolution, G.E. Hill and K.J. McGraw, eds. (Cambridge, MA: Harvard University Press), pp. 87-136. 
166 8 9

80 81 82

Figure 1. Male house sparrows used in the experiment showing $(A)$ the initial bib just before the beginning of the experiment, $(B)$ the bib after all its feathers and neighboring feathers were plucked in the beginning of the experiment, and $(\mathrm{C})$ the final bib with newly grown feathers at the end of the experiment.

Figure 2. Relationship between bib size and pheomelanin levels in bib feathers (TTCA levels) of male house sparrows. The ordinate axis depicts the residual size of the bib patch, calculated as the residuals of a GLM with bib size as the response variable, treatment as a fixed factor and tarsus length as a covariate, but not including TTCA levels. Thus, the ordinate axis values shows the variation in bib patch size that is not explained by neither treatment nor tarsus length so that the association with TTCA levels is not affected by those confounding factors. The line is the regression line. 
190

191

192

193

194

195

196

197

198

199

200

201

202

203

204

205

206

207

208

209

210

$211 \quad$ Figure 1

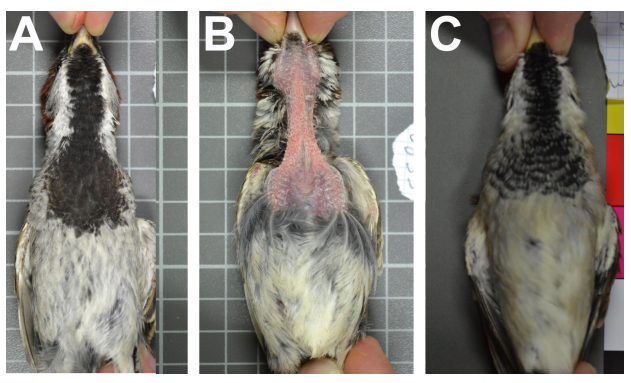




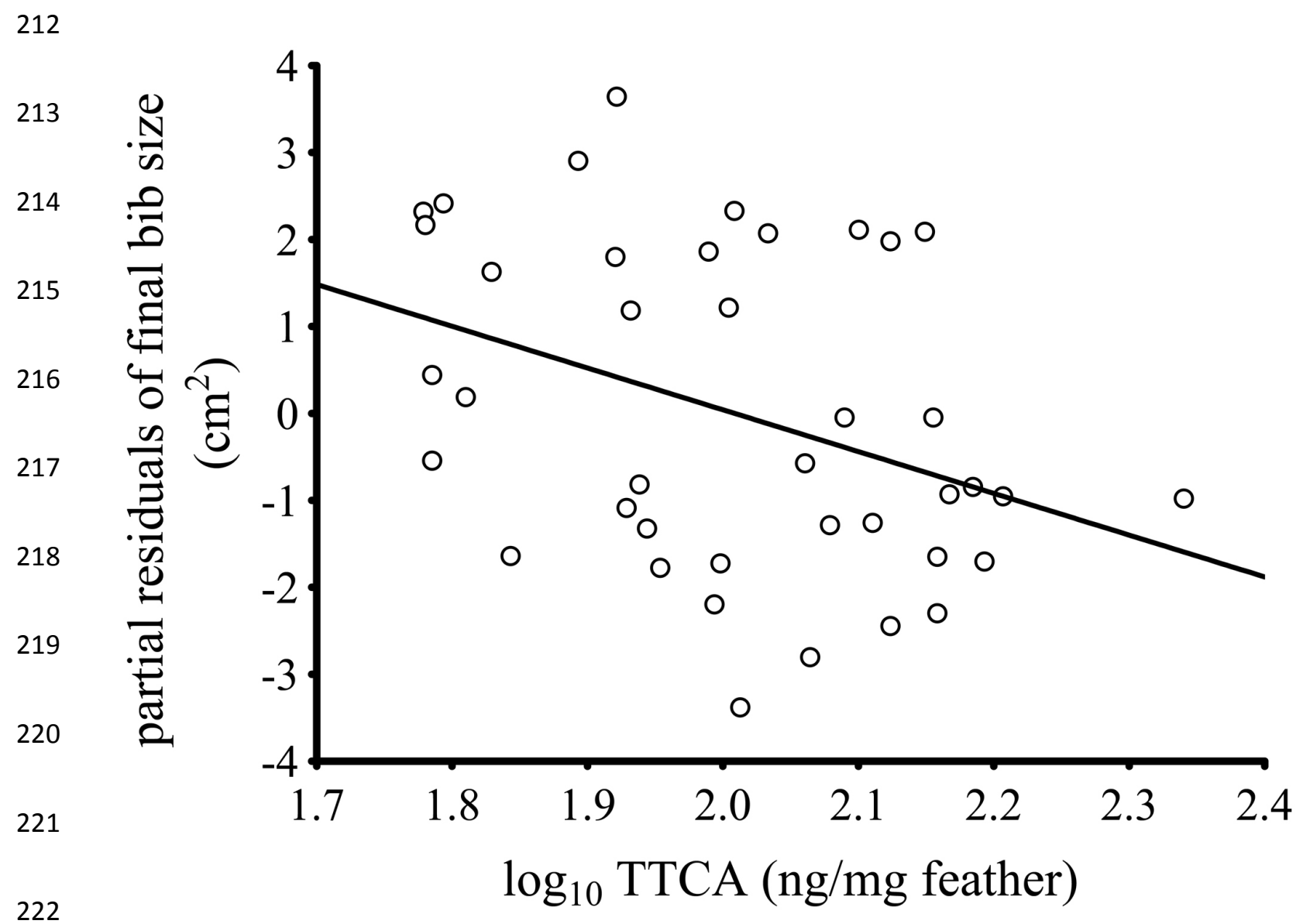

$223 \quad$ Figure 2

224

225

226

227

228

229

230

231

232 
Supporting information for:

\title{
Black bib size is associated with feather content of pheomelanin in male
} house sparrows

\author{
Ismael Galván, Kazumasa Wakamatsu and Carlos Alonso-Alvarez
}

\section{Experimental procedure}

In June-July 2010, 53 free ranging adult male house sparrows were captured with mist nests in the surroundings of the Dehesa Galiana experimental facility (Ciudad Real, Spain), identified with a metal ring and housed alone in individual cages ( $0.6 \mathrm{~m}$ long $\times 0.4 \mathrm{~m}$ wide $\times 0.4 \mathrm{~m}$ tall; Italgabbie, Caltrano, Italy) in an indoor aviary $(7.4 \times 3.3 \times 2.5 \mathrm{~m})$. The birds were provided with ad libitum water and food for canaries and cuttlefish bone. On 13 July, tarsus length was measured with digital calipers. This process was made for the aims of an experiment in which the levels of glutathione (GSH), a key intracellular antioxidant, were manipulated in the birds.

In the house sparrow, the moulting period takes place at the time our experiment was conducted (i.e. July-September; Anderson, 2006), but to avoid inter-individual differences in the phenology of plumage moult, on 20 July we plucked the feathers of the bib patch, thus inducing the growth of all the bib feathers. The bib area of male house sparrows is fixed at moult and does not change until the next molt (Anderson, 2006), but bib feathers lose their grey tips during the course of the season so that bibs at the end of the season have lost part of feather barbules. Thus, to analyse the association between bib size and melanin content we only considered the size of newly developed bibs (figure 1). With this aim, on 27 September the birds were captured and a photograph of the bib of birds was taken with a digital camera (Olympus E-50). The birds were held in the same posture, at a fixed distance from the camera, under standardized illumination (figure 1). Bib size was measured by selecting the total black area on throat and chest with Adobe Photoshop and converting pixels to $\mathrm{cm}^{2}$ with the reference of a scale that was included as background in all photographs. The analysis of bib size was made by a technician blinded to the aims of the study. The body mass of birds was measured with a digital balance. We also plucked $15-20$ feathers at random from the inner part of the bib of all birds, which were stored in plastic bags and kept at dark until 

biochemical analyses were carried out. The birds were released back to nature at the same site where they were captured after all measurements were taken.

\section{Analysis of melanins}

The analysis of melanins was based on the formation and detection by HPLC of specific degradation products, 4-amino-3-hydroxyphenylalanine (4-AHP) by reductive hydrolysis of pheomelanin with hydriodic acid (HI) and pyrrole-2,3,5-tricarboxylic acid (PTCA) and thiazole-2,4,5-tricarboxylic acid (TTCA) by alkaline $\mathrm{H}_{2} \mathrm{O}_{2}$ oxidation of eumelanin and pheomelanin, respectively. Thus, 4-AHP and TTCA are specific to pheomelanin and PTCA is specific to eumelanin. We used two alternative markers for pheomelanin because TTCA and 4-AHP are specific of the two different moieties of the pigment, benzothiazole and benzothiazine, respectively (Wakamatsu et al., 2009). Details of sample analyses have been published elsewhere (e.g., Galván et al. 2012).

\section{Statistical analyses}

General linear models (GLM) were used to analyse the association between bib size and the levels of pheomelanin and eumelanin in feathers. Bib size was the response variable, and pheomelanin (TTCA or 4AHP) or eumelanin (PTCA) levels were added as covariates ( $\log _{10}$-transformed). Given the level of correlation between the different melanin markers (Pearson correlation tests: PTCA-TTCA: $r=0.34, p=$ 0.033; PTCA-4-AHP: $r=-0.04, p=0.827$; TTCA-4-AHP: $r=0.68, p<0.0001$, all $n=40$ ), the effect of each melanin marker on bib size was tested on separate models. Variation in body size was controlled for by adding tarsus length as an additional covariate. As birds were exposed to an experimental treatment, bib size may be affected by this potential source of variation, so treatment (control vs. manipulated) was added to the model as a fixed factor as well as its interaction with melanin level. A backward stepwise procedure was used to remove non-significant terms, using a $p$-value of 0.1 as a threshold to abandon the model.

We also calculated the total content of eumelanin and pheomelanin of the bib of house sparrows by multiplying the concentrations of TTCA, 4-AHP and PTCA by bib size, as well as the coefficient of variation (CV, calculated with values in $\left.\log _{10}\right)$ to determine the variability among individual birds in the total content of the different melanin forms. 


\section{Results}

291

292

293

The mean $( \pm$ SE) concentration of TTCA (the benzothiazole unit marker for pheomelanin) in the bib feathers of male house sparrows was $65.69 \pm 7.77 \mathrm{ng} / \mathrm{mg}$ feather, while the mean concentration of 4-AHP (the benzothiazine unit marker for pheomelanin) was $23.66 \pm 2.70 \mathrm{ng} / \mathrm{mg}$ feather. The mean concentration of PTCA (eumelanin marker) was $1072.75 \pm 35.57 \mathrm{ng} / \mathrm{mg}$ feather.

The mean $( \pm$ SE) total content of pheomelanin was $665.51 \pm 38.60 \mathrm{ng}$ with a CV of 5.85 for TTCA, and $324.23 \pm 51.65 \mathrm{ng}$ with a CV of 10.85 for 4-AHP. Regarding eumelanin, the mean total content of PTCA was $6570.31 \pm 405.11 \mathrm{ng}$ and its CV was 4.81 .

The association between bib size and TTCA levels did not change when only control birds were considered $\left(b=-8.08, t=-2.19, F_{1,17}=4.81, p=0.042\right)$. All results kept unchanged if tarsus length was removed from the models.

\section{Discussion}

The dispersion of data around the mean was higher for the total content of pheomelanin in the bib than in the total content of eumelanin. Thus, although the total content of eumelanin in the bib was considerably greater than the total content of pheomelanin, the content of pheomelanin was more variable among individual birds than the content of eumelanin, up to more than twice in the case of 4-AHP. This supports the idea that pheomelanin, even at relative low concentrations in the bib of house sparrows, may have a significant role mediating the honesty in the bib size of male house sparrows. This is because honest signals are traits whose expression depends on the physical condition of the bearers and are sexually selected (which applies to the bib of male house sparrows; Nakagawa et al., 2007), and this makes that these traits are a large target for genetic variation (Tomkins et al., 2004).

\section{References}

Anderson, T.R. 2006. Biology of the ubiquitous house sparrow (New York: Oxford University Press). 
315 Galván, I., Erritzøe, J., Wakamatsu, K. and Møller, A.P. (2012). High prevalence of cataracts in birds with pheomelanin-based colouration. Comp. Biochem. Physiol. A 162, 259-264.

317 Nakagawa, S., Ockendon, N., Gillespie, D.O.S., Hatchwell, B.J. and Burke, T. (2007). Assessing the function of house sparrows' bib size using a flexible meta-analysis method. Behav. Ecol. 18, 831-840.

319 Tomkins, J.L., Radwan, J., Kotiaho, J.S. and Tregenza, T. (2004). Genic capture and resolving the lek paradox. Trends. Ecol. Evol. 19, 323-328.

321 Wakamatsu, K., Ohtara, K. and Ito, S. (2009). Chemical analysis of late stages of pheomelanogenesis: conversion of dihydrobenzothiazine to a benzothiazole structure. Pigment Cell Melanoma Res. 22, 474-486. 